than that of a tuberculin skin test not confounded by Bacille Calmette-Guérin vaccination or nontuberculous mycobacteria. This must be determined by ongoing prospective restriction fragment length polymorphism studies observing the clinical course of QuantiFERON-gold-positive contact persons. These use "clustering" with the source case and observe who becomes infected with the disease. As QuantiFERON-gold is only just being implemented for routine use in western industrial countries, this question is not sufficiently answered. In our view, it would be speculative to distinguish between the predictive values of the two testing procedures. Therefore, we assigned a uniform probability of re-activation to manifest tuberculosis disease to subjects testing positive to either the tuberculin skin test or the QuantiFERON-gold assay.

\section{R. Diel}

Heinrich-Heine-University, Postfach 101007, Düsseldorf 40001, Germany.

\section{REFERENCES}

1 Diel R, Nienhaw A, Lange C, Schaberg T. Cost-optimisation of screening for latent tuberculosis in close contacts. Eur Respir J 2006; 28: 35-44.

2 Behr A, Hopewell PC, Paz EA, Kamamura LM, Schecter GF, Small PM. Predictive value of contact investigation for identifying recent transmission of Mycobacterium tuberculosis. Am J Respir Crit Care Med 1998; 158: 465-469.

3 Diel R, Schneider S, Meywald-Walter K, Ruf CM, RüschGerdes S, Niemann S. Epidemiology of tuberculosis in Hamburg, Germany: long-term population-based analysis applying classical and molecular epidemiological techniques. J Clin Microbiol 2002; 40: 532-539.

4 Diel R, Meywald-Walter K, Gottschalk R, Rüsch-Gerdes S, Niemann S. Ongoing outbreak of tuberculosis in a lowincidence community: a molecular-epidemiological evaluation. Int J Tuberc Lung Dis 2004; 8: 855-861.

\title{
Therapy of exophytic bronchial tumorous stenosis by flexible cryoprobe
}

\section{To the Editors:}

We read with interest the article by VERGNON et al. [1] in the European Respiratory Journal, about the place of cryotherapy, brachytherapy and photodynamic therapy in the therapeutic bronchoscopy of lung cancers. The article describes indications and limitations of cryotherapy. In the opinion of VERGNON et al. [1], the effect of cryotherapy is delayed and is, therefore, not useful for the immediate debulking of an obstructive endoluminal bronchial tumour. For these cases, VERGNON et al. [1] advise coring of the tumour with the rigid bronchoscope and subsequent laser therapy or electrocautery. The indication for cryotherapy in this setting is seen as a treatment option for any remaining infiltrative parts of the tumour into the bronchial wall, but not as a treatment option of first choice to remove the main part of an exophytic tumour.

In our clinic, we have positive experience in a large number of patients with the application of cryotherapy for the immediate treatment of acute dyspnoea in patients with obstructive malignant bronchial tumours. We use the cryorecanalisation technique with the rigid bronchoscope under general anaesthesia, as well as with a flexible technique during local anaesthesia and sedation. The commercially available flexible cryoprobe (ERBE, Tübingen, Germany) we use is $780 \mathrm{~mm}$ in length and $2.3 \mathrm{~mm}$ in diameter. The probe's tip is cooled to $-89.5^{\circ} \mathrm{C}$ by sudden gas decompression (nitrous oxide) in the probe's head. The cryoprobe is a closed system, which can be re-sterilised and re-used. In many cases, we use the cryorecanalisation method in combination with argon plasma coagulation for a more immediate treatment of diffuse tumour bleeding after coring with the rigid bronchoscope.
We apply the following technique: 1) the flexible cryoprobe is introduced via a flexible bronchoscope in combination with an Ikeda tubus or a rigid bronchoscope; 2) the tip of the cryoprobe is pushed into the protruding exophytic tumour mass and freezing is started for $\leqslant 5 \mathrm{~s}$ with a footpad; 3 ) the cryoprobe, including the flexible bronchoscope, is abruptly mechanically removed, a tumour mass of 3-5 $\mathrm{mm}$ is frozen at the tip of the cryoprobe and can easily be extracted; 4) rapid thawing is induced in a water bath for $\leqslant 5 \mathrm{~s}$; and 5) the procedure is repeated until the tumour mass has been extracted and the target bronchus is re-opened. If necessary, subsequent stent implantation is performed in the same session without any problems. With this technique, we have seen no severe bleedings if coagulation parameters were within the normal range. Overall, we have a very low complication rate. Severe bleeding during re-canalisation with the cryoprobe is very rare, we have never seen perforations, and the primary success rate of re-canalisation is very high $(>90 \%)$, even in longdistance stenoses, and in our experience comparable to that of laser beam or electrocautery. The feasibility of this method for the immediate management of acute airway obstruction has already been published [2]. In contrast to the cryotherapy technique described by VERGNON et al. [1], which requires a second bronchoscopy after 8-10 days for the removal of necrotic tissue, a second-look bronchoscopy is not mandatory with our technique.

In addition, the specimens obtained by cryorecanalisation for diagnostic purposes are much larger and of excellent tissue quality, without squeezing artefacts, compared with specimens extracted using forceps. In a study with 60 patients, comparing 
specimen quality in forceps biopsy and cryobiopsy, we found the cryobiopsy technique to have a superior diagnostic quality [3].

In summary, the use of the flexible cryoprobe for mechanical removal of obstructive exophytic bronchial tumours is a good alternative to laser therapy or electrocautery in planned bronchoscopic interventions as well as in patients with acute dyspnoea and tumorous bronchial obstruction. Cryorecanalisation is safe, cheap and readily applicable with an immediate success and relief of symptoms.

\section{Schumann, C. Kropf, T. Wibmer, T. Merk and S. Krüger Dept of Internal Medicine II, University of Ulm, Ulm, Germany.}

\section{REFERENCES}

1 Vergnon JM, Huber RM, Moghissi K. Place of cryotherapy, brachytherapy and photodynamic therapy in therapeutic bronchoscopy of lung cancers. Eur Respir J 2006; 28: 200-218.

2 Hetzel M, Hetzel J, Schumann C, Marx N, Babiak A. Cryorecanalization: a new approach for the immediate management of acute airway obstruction. J Thorac Cardiovasc Surg 2004; 127: 1427-1431.

3 Schumann C, Mattfeldt T, Hetzel M, Hetzel J, Lepper P. Improving the diagnostic yield of endobronchial biopsies by flexible cryoprobe in lung cancer - comparison of forceps and cryoprobe technique. Eur Respir J 2004; 24: Suppl. 48, 491s.

DOI: $10.1183 / 09031936.00095106$

\section{From the authors:}

I have read with great attention and interest the letter and comments by $\mathrm{C}$. Schumann and co-workers about our review on cryotherapy, brachytherapy and photodynamic therapy, which was recently published in the European Respiratory Journal [1].

All studies into the relationships between cold and tissues underline the delayed action of cold: delayed vascular thrombosis then delayed necrosis. This point explains the delayed action of cryotherapy. The unique described immediate interaction between cold and tissue is the glue effect, which allows the extraction of foreign bodies. C. Schumann and coworkers have presented good studies on the use of newly designed flexible cryoprobes for obtaining large biopsies during flexible bronchoscopies. One of these studies was indicated in our paper [2]. Hetzel et al. [3] also used this technique to perform immediate re-canalisation in cases of bulky tumours. We know this technique and its results well, and have shared discussion with the authors on several occasions about their experiences. However, it is true that in our paper we did not speak about this experience, and we would like to thank $\mathrm{C}$. Schumann and co-workers for detailing their technique.

In our paper, these results were not reported for two main reasons. First, the aim of the paper was to review the methods of therapeutic endoscopy of delayed action, which explains why immediate action of these methods, including cryotherapy, was not detailed. The second reason is that the technique proposed by $\mathrm{C}$. Schumann and co-workers is extremely original. In fact, to my knowledge, this method is not currently being used around the world. Therefore, we must consider that this new technique is in progress and further reports are needed. Haemorrhagic risks should be carefully analysed. Vessel thrombosis induced by cold is delayed by $\sim 8 \mathrm{~h}$. With immediate cryo-extraction proposed in this new technique, there is no possible coagulation and this method is closed to simple mechanical resection. Mechanical resection is widely used with a rigid bronchoscope where bleeding is easily controlled, but, with a flexible bronchoscope, this point could be crucial.

I hope that further papers by C. Schumann and co-workers will quickly confirm these preliminary results and give a new impulse for utilising cryotherapy.

\section{J-M. Vergnon}

Dept of Chest Diseases and Thoracic Oncology, University Hospital of Saint Etienne, Hôpital Nord, St Etienne, France.

\section{REFERENCES}

1 Vergnon JM, Huber RM, Moghissi K. Place of cryotherapy brachytherapy and photodynamic therapy in therapeutic bronchoscopy of lung cancers. Eur Respir J 2006; 28: 200-218.

2 Schumann C, Mattfeldt T, Hetzel M, Hetzel J, Lepper PM. Improving the diagnostic yield of endobronchial biopsies by flexible cryoprobe in lung cancer-comparison of forceps and cryoprobe technique. Eur Respir J 2004; 24: Suppl. 48, S491.

3 Hetzel M, Hetzel J, Schumann C, Marx N, Babiak A. Cryorecanalization: a new approach for a immediate management of acute airway obstruction. I Thorac Cardiovasc Surg 2004; 127: 1427-1431.

DOI: $10.1183 / 09031936.00114106$ 